\title{
Discordance rate of HER2 status in primary breast carcinomas versus synchronous axillary lymph node metastases: a multicenter retrospective investigation
}

\author{
This article was published in the following Dove Press journal: \\ OncoTargets and Therapy \\ II July 2014 \\ Number of times this article has been viewed
}

\author{
Antonio leni' \\ Valeria Barresi ${ }^{\prime}$ \\ Rosario Caltabiano ${ }^{2}$ \\ Anna Maria Cascone ${ }^{3}$ \\ Rachele Del Sordo ${ }^{4}$ \\ Daniela Cabibi ${ }^{5}$ \\ Pio Zeppa ${ }^{3}$ \\ Salvatore Lanzafame ${ }^{2}$ \\ Angelo Sidoni ${ }^{4}$ \\ Vito Franco ${ }^{5}$ \\ Giovanni Tuccari' \\ 'Department of Human Pathology \\ Gaetano Barresi, Section of \\ Anatomic Pathology, University \\ of Messina, Messina, ${ }^{2}$ Department \\ GF Ingrassia, Section of Anatomic \\ Pathology, University of Catania, \\ Catania, ${ }^{3}$ Department of Medicine \\ and Surgery, University of \\ Salerno, Salerno, ${ }^{4}$ Institute of \\ Pathologic Anatomy and Histology, \\ Division of Cancer Research, \\ University of Perugia, Perugia, \\ ${ }^{5}$ Department of Human Pathology, \\ Section of Anatomic Pathology, \\ University of Palermo, Palermo, Italy
}

Correspondence: Giovanni Tuccari Department of Human Pathology Gaetano Barresi, AOU Polyclinic G Martino, Via Consolare Valeria I,

Messina 98125, Italy

$\mathrm{Tel}+39902212539$

$\mathrm{Fax}+39902928150$

Email tuccari@unime.it
Background: Human epidermal growth factor receptor 2 (HER2) is considered to be a therapeutic and prognostic marker in the management of breast carcinoma (BC), although discordance rates between primary and metastatic or locally recurrent lesions have been reported.

Methods: One hundred and forty-eight paraffin-embedded BC tissues from patients of mean age 59.27 (33-96) years and corresponding synchronous lymph node metastases were collected and retrospectively studied using immunohistochemistry and fluorescence in situ hybridization to evaluate HER2 status. Fleiss-Cohen weighted $\mathrm{k}$ statistics were used to assess the concordance rate between HER2 status of the primary BC and the synchronous metastatic lesions.

Results: The overall concordance rate for HER2 was 95.28\%. Eighty-nine cases were concordantly HER2-negative in primary BC and nodal metastases, and 52 cases were HER2-positive in both primary and metastatic tumors. Changes in HER 2 status between primary BC and corresponding synchronous metastases were observed in seven (4.72\%) cases. Three of the discordant cases were HER2-negative in the primary tumor and HER2-positive in the metastases, while four cases were HER2-positive in the primary BC and HER2-negative in the metastases. No significant correlations were identified between HER2 status and expression of hormone receptors, growth fraction (Ki-67), or other histopathological parameters (pT, pN, grade).

Conclusion: Simultaneous determination of HER2 in BC and corresponding metastatic lymph nodes is not mandatory, but may strongly influence the therapeutic management. It was demonstrated that loss of HER2 amplification results in worse post-relapse survival and overall survival in $\mathrm{BC}$ patients and, on the other hand, a gain in HER2 expression in metastatic lymph nodes of BC may allow the possibility of a targeted treatment. Thus, our opinion is that significant prognostic information may be obtained by simultaneous assessment of HER2 status in both primary and synchronous metastatic BC.

Keywords: human epidermal growth factor receptor 2, breast cancer, synchronous lymph nodes, metastases, prognosis

\section{Introduction}

Assessment of human epidermal growth factor receptor 2 (HER2) status in patients with breast carcinoma $(\mathrm{BC})$ is commonly performed using neoplastic tissue from the primary tumor. However, in recent years, several papers have shown that HER2 status may be different in metastatic lesions when compared with the primary tumor, ${ }^{1-6}$ and this discrepancy is more frequently found in distant metastases than in locoregional ones. ${ }^{7,8}$ Discordance in HER2 status may be found not only between primary BC and its metastases, but also between consecutive relapses of the same tumor, with similar 
proportions of cases turning from negative to positive and vice versa, with such a change occurring mainly in second or subsequent progressions. ${ }^{9}$

The possible divergence in HER2 status between the primary BC and its metastases, or in consecutive metastases of the same tumor, is significant because it may modify a patient's sensitivity to targeted therapy, ${ }^{6}$ which might be appropriate for the primary tumor but not for metastases and vice versa. ${ }^{10-13}$ For this reason, some authors have suggested that HER2 status should be reassessed in metastatic $\mathrm{BC}$ tumor tissue to establish if a particular therapy is actually appropriate. ${ }^{1,4-6,12,14}$

The reasons underlying changes in HER2 status in metastases when compared with the primary BC have not yet been clarified. Nonetheless, in most of the studies, primary BC was compared with recurrent or metachronous lesions. . $^{2,4,12,13,15-18}$ Discordance between primary and metachronous tumors may occur for a variety of reasons, including variability in technical assessment or preanalytical factors, such as use of different fixation intervals and procedures, ${ }^{19-21}$ or biological modifications during tumor progression. In addition, if the patient undergoes chemotherapy, metachronous tumors may show polyploidization in tumor cells as a treatment-related effect, which may be misinterpreted as HER2 amplification, with the potential for false positive results. ${ }^{22}$

In the comparison between primary tumor and paired synchronous lymph node metastases, the possibility that variability in HER2 status might depend on external factors is reduced, as both the specimens may be collected during the same surgical procedure, prior to any influence of therapy, and submitted to the same fixation methodology. Thus, analysis of the concordance rate between primary $\mathrm{BC}$ and synchronous metastases could provide more information on the biological processes underlying changes in HER2 status. However, to the best of our knowledge, the few studies that have investigated modification of HER2 status in paired samples of primary BC and synchronous node metastases were only performed using tissue microarrays. . 23,24 Thus, in order to minimize the probability of technical sampling errors due to the tissue microarray approach, in the present study we retrospectively analyzed HER2 variation in a cohort of primary BC and synchronous nodal metastases by using whole sections of the tumors.

\section{Materials and methods}

One hundred and forty-eight surgical BC specimens from patients of mean age 59.27 (33-96) years and corresponding synchronous lymph node metastases were retrospectively collected from five separate anatomic pathology units in Italy.
None of the patients had received neoadjuvant chemotherapy or other therapies prior to surgical resection.

Data on the histotype, grading, hormone receptor expression, growth fraction (Ki67 labelling index), and HER2 status were available in all cases. Personal patient details were nonidentifiable and all patients had provided written consent to their medical information being used for research purposes, in accordance with the Declaration of Helsinki. Approval for this retrospective study was obtained from the local ethics committees of the universities of Messina, Catania, Salerno, Perugia, and Palermo, Italy.

For each case, $4 \mu \mathrm{m}$-thick sections from the formalinfixed paraffin-embedded representative tissue blocks of primary tumor and nodal metastases were set for HER2 immunohistochemistry. HER2 expression was investigated in all the nodal metastases; at least three metastatic lymph nodes were tested for each case.

The immunohistochemical analysis for evaluation of HER2 status was carried out using the HercepTest ${ }^{\mathrm{TM}}$ kit (Dako, Glostrup, Denmark) with an automated procedure (Autostainer Link 48, Dako), according to the manufacturer's instructions. The same procedure was applied at all of the units, with HER2 positivity defined as $3+$ when strong membranous staining was noted in at least $30 \%$ of cells, $2+$ when weak to moderate complete membranous staining was evident in $10 \%-30 \%$ of tumor cells, $1+$ when a faint or weak and incomplete membrane staining was observed, and 0 when no staining was observed or when staining was present in less than $10 \%$ of neoplastic cells. Fluorescence in situ hybridization (FISH) analysis was performed using a HER2 FISH PharmDx ${ }^{\mathrm{TM}}$ kit (Dako) in cases showing 2+ immunostaining, and gene amplification was recorded when the HER2/centromeric probe for chromosome 17 signal ratio was $\geq 2$.0. In order to avoid significant interobserver variability in HER2 immunohistochemical scoring, we selected a majority $(140 / 148)$ of specimens with an easily attributable score, such as $3+(56 / 148)$ or $0(84 / 148)$; in fact, these latter two categories represent the more reproducible scores in $\mathrm{BC}$, the first certainly positive and the second certainly negative, that may greatly limit the interobserver judgment impair. After a first assessment in each unit of pathology, all the immunostained slides were reassessed, in a random order, by two observers (AI and GT), blinded of previous paired data. In the event of disagreement, cases were jointly discussed using a doubleheaded microscope until agreement was reached.

Fleiss-Cohen weighted k statistics were used to assess concordance between the HER2 status of the primary BC and metastatic synchronous lesions; $\mathrm{k}$ values of $0-0.2$ were 
regarded as no agreement, $0.21-0.4$ as fair agreement, $0.41-0.6$ as moderate agreement, $0.61-0.8$ as substantial agreement, and $0.81-1$ as almost perfect agreement. Statistical correlations between HER2 status and the other histopathological parameters were investigated using the chi-squared test. A $P$-value $<0.05$ was considered to be statistically significant. The data were analyzed using Statistical Package for the Social Sciences version 6.1.3 software (SPSS Inc., Chicago, IL, USA).

\section{Results}

The histopathological characteristics and immunohistochemistry of cases included in the study are summarized in Table 1. With reference to the histotype, all primary BCs had been diagnosed as ductal invasive carcinomas, with the following grading: five G1 (3.39\%), $65 \mathrm{G} 2$ (43.91\%), and $78 \mathrm{G} 3$ (52.70\%). According to pTNM staging, 56 cases had been classified as pT1 (37.83\%), 62 as pT2 (41.89\%), 17 as pT3 (11.48\%), and 13 as pT4 (8.80\%), while N status was N1 in 58 cases $(39.18 \%)$, N2 in $60(40.55 \%)$ and N3 in $30(20.27 \%)$.

With regard to HER2 immunohistochemistry, 56 (37.84\%) primary BCs had a score of $3+, 84(56.76 \%)$ scored 0 (no staining), while six (4.05\%) were scored as $2+$ and two
$(1.35 \%)$ as $1+$. FISH analysis revealed no amplification in all cases scored as $2+$. FISH was carried out in both the $1+$ cases since these carcinomas showed high-grade, high Ki67 value, $\mathrm{N}+$ status, and an absence of endocrine receptor expression, but no HER2 amplification was identified.

Thus, among the primary BCs, HER2 was considered as amplified in 56 cases (37.84\%) and not amplified in 92 cases $(62.16 \%)$, the latter representing the total number of cases scored as $0,1+$, and $2+$, all of which were FISH-negative (Table 1). When the synchronous nodal metastases were considered, HER2 was scored as 3+ in 55 cases, while 93 cases were HER2-negative. No gene amplification was found when FISH analysis was carried out in the $1+$ tumors. The $\mathrm{k}$ value for concordance in HER2 status between the primary tumors and metastases was 0.87 (almost perfect agreement).

The overall concordance rate was $95.28 \%$; specifically, 89 cases were concordantly HER2-negative in primary BC and in nodal metastases, while 52 cases were HER2-positive in both primary and metastatic tumors (Table 1). Changes in HER2 status between primary $\mathrm{BC}$ and corresponding synchronous metastases were evident in seven (4.72\%) cases (Table 2). Three of the discordant cases were HER2-negative in the primary tumor and positive in the metastases, while four cases were HER2-positive

Table I Histopathological characteristics and biomolecular phenotypes of primary breast cancer and lymph node metastases

\begin{tabular}{|c|c|c|c|c|c|c|c|c|c|}
\hline \multirow[t]{2}{*}{ Parameter } & \multirow{2}{*}{$\begin{array}{l}\text { Cases } \\
\text { (n) }\end{array}$} & \multicolumn{4}{|c|}{ HER2+ tumors } & \multicolumn{4}{|c|}{ HER2- tumors } \\
\hline & & $\mathbf{n}$ & $\begin{array}{l}\text { Node } \\
\text { concordance } \\
(\mathrm{T} / \mathrm{N}=+/+)\end{array}$ & $\begin{array}{l}\text { Node } \\
\text { discordance } \\
(\mathrm{T} / \mathrm{N}=+/-)\end{array}$ & $P$-values & $\mathbf{n}$ & $\begin{array}{l}\text { Node } \\
\text { concordance } \\
(\mathrm{T} / \mathrm{N}=-l-)\end{array}$ & $\begin{array}{l}\text { Node } \\
\text { discordance } \\
(\mathrm{T} / \mathrm{N}=-l+)\end{array}$ & $P$-values \\
\hline HER2 status & 148 & 56 & $52(92.9 \%)$ & $4(7.1 \%)$ & - & 92 & 89 (96.7\%) & $3(3.3 \%)$ & - \\
\hline $\mathrm{pT}$ & & & & & 0.774 & & & & 0.619 \\
\hline TI & 56 & 20 & 19 & 1 & & 37 & 36 & 1 & \\
\hline $\mathrm{T} 2$ & 62 & 24 & 22 & 2 & & 37 & 36 & 1 & \\
\hline T3 & 17 & 7 & 6 & 1 & & 10 & 9 & 1 & \\
\hline T4 & 13 & 5 & 5 & 0 & & 8 & 8 & 0 & \\
\hline $\mathrm{pN}$ & & & & & 0.815 & & & & 0.545 \\
\hline NI & 58 & 22 & 21 & 1 & & 36 & 34 & 2 & \\
\hline N2 & 60 & 21 & 19 & 2 & & 40 & 39 & 1 & \\
\hline N3 & 30 & 13 & 12 & 1 & & 16 & 16 & 0 & \\
\hline Grade & & & & & $0.48 I$ & & & & 0.690 \\
\hline GI & 5 & 0 & 0 & 0 & & 5 & 5 & 0 & \\
\hline $\mathrm{G} 2$ & 65 & 19 & 17 & 2 & & 47 & 46 & 1 & \\
\hline G3 & 78 & 37 & 35 & 2 & & 40 & 38 & 2 & \\
\hline ER status & & & & & 0.335 & & & & 0.496 \\
\hline ER+ & 108 & 29 & 26 & 3 & & 80 & 77 & 3 & \\
\hline ER- & 40 & 27 & 26 & 1 & & 12 & 12 & 0 & \\
\hline PR status & & & & & 0.153 & & & & 0.475 \\
\hline $\mathrm{PR}+$ & 100 & 23 & 20 & 3 & & 79 & 76 & 3 & \\
\hline PR- & 48 & 33 & 32 & 1 & & 13 & 13 & 0 & \\
\hline $\mathrm{Ki}-67$ & & & & & 0.397 & & & & 0.747 \\
\hline $\mathrm{KI}-67 \leq 14 \%$ & 46 & 8 & 8 & 0 & & 39 & 38 & 1 & \\
\hline $\mathrm{KI}-67>14 \%$ & 102 & 48 & 44 & 4 & & 53 & 51 & 2 & \\
\hline
\end{tabular}

Abbreviations: ER, estrogen receptor; PR, progesterone receptor; HER2, human epidermal growth factor receptor 2; pT, post-surgical tumor size; pN, post-surgical node status. 
Table 2 Seven cases with changes in HER2 status between the primary $\mathrm{BC}$ and corresponding synchronous nodal metastases

\begin{tabular}{llllllll}
\hline pT & pN & Grading & ER\% & PR\% & $\begin{array}{l}\text { Ki-67\% } \\
\text { HER2 } \\
\text { primary } \\
\text { lesions }\end{array}$ & $\begin{array}{l}\text { HER2 } \\
\text { metastatic } \\
\text { lesions }\end{array}$ \\
\hline T2 & N3 & G3 & 90 & 15 & 20 & $3+$ & 0 \\
T3 & N2 & G2 & 25 & 70 & 15 & $3+$ & 0 \\
T2 & N2 & G3 & 0 & 0 & 30 & $3+$ & 0 \\
TIc & N1 & G2 & 80 & 80 & 35 & $3+$ & I+* \\
TIc & N1 & G3 & 90 & 30 & 15 & 0 & $3+$ \\
T2 & N2 & G3 & 0 & 0 & 35 & I+* & $3+$ \\
T3 & N1 & G2 & 0 & 0 & 20 & 0 & $3+$ \\
\hline
\end{tabular}

Notes: *Not amplified by fluorescence in situ hybridization. TIc is a variant of PTI tumours, with a neoplastic dimension ranging from 1.5 to $2.0 \mathrm{~cm}$.

Abbreviations: ER, estrogen receptor; PR, progesterone receptor; HER2, human epidermal growth factor receptor 2; $\mathrm{pT}$, post-surgical tumor size; $\mathrm{pN}$, post-surgical node status; $\mathrm{BC}$, breast carcinoma.

in the primary BC and HER2-negative in the metastases. No significant correlations emerged between HER2 status and expression of hormone receptors, growth fraction (Ki-67), or other histopathological parameters (pT, $\mathrm{pN}$, grade).

\section{Discussion}

Although HER2 status is generally considered to be stable during the metastatic process, with a high concordance rate between primary tumors and metastases, many reports have shown a clinically significant HER 2 discordance, ranging between $0 \%$ and $34 \%$, when comparing primary and recurrent or metastatic BC. ${ }^{1,2,4,12,13,15-18,25,26}$ The reported modification in HER2 status is from positive to negative, but has also been reported in the opposite direction, ${ }^{2}$ although the prevalence of HER2-negative conversion outnumbers that of HER2-positive conversion. ${ }^{4} \mathrm{Of}$ note is that the analyses concerning HER2 changes have been preferentially performed in recurrent locoregional or metachronous breast lesions, ${ }^{2,4,12,13,15-18}$ while a quantitative analysis of HER2 modifications in small cohorts of paired primary BC and synchronous node metastases were exclusively performed on tissue microarrays, with conflicting reports. ${ }^{1,23-25}$ For this reason, in the present study, we retrospectively analyzed whole sections of original breast tumor and metastatic lymph node tissue, with a conspicuous reduction of error in the technical sampling as occurs by tissue microarray approach. Moreover, the use of paired samples of BC and lymph nodes collected during the same surgical procedure and subjected to the same fixation methodology avoided technical variability and therefore, immunohistochemical results, being always reproducible in primary and metastatic lesions, may not determine any unjustified change in the targeted therapeutic management.

It has been stressed that higher discordance is found using immunohistochemistry for the determination of HER2 status than when using FISH, suggesting that methodology may play a significant role in the discordance. ${ }^{2,26}$ Indeed, when the same tumors were tested by both immunohistochemistry and FISH, the discordance rate was $18 \%$ using immunohistochemistry and $12 \%$ by FISH. ${ }^{13,27}$ In our opinion, this discrepancy could be minimized if only $3+$ scored or FISH + cases are considered as amplified for HER2, since this could avoid the need for a central review of the specimens, as has been proposed previously. ${ }^{28} \mathrm{Hence}$, for the present analysis, we mainly selected frankly positive $(3+)$ or negative (0) cases, since this may reduce the bias connected with the reproducibility of HER2 determination. Indeed, when all the cases were finally reassessed by two observers blinded to the previous paired data, the only disagreement that arose involved cases showing a $2+$ or $1+$ score that were tested by FISH. Thus, we may suppose that the HER 2 discordance rate of $4.72 \%$ between the primary $\mathrm{BC}$ and their synchronous nodal metastases could not be due to doubtful HER2 assessment. In accordance with the relevant literature, we found both positive and negative conversion of HER2 status between the primary tumor and the lymph node metastases. ${ }^{1,25,28,29}$

Our discordance rate was lower than that reported for locoregional recurrences and metachronous metastases, $2,4,13,15,16,18$ but quite similar to the figure of $3.4 \%$ reported for metastatic lymph nodes by tissue microarrays, ${ }^{23}$ although other recent studies have shown very different discordance rates for HER2 expression, ranging between $0 \%$ and $9 \%$ in archival paired tissue microarray material. ${ }^{23,30}$

The conversion in HER2 status is consistent with the evidence that HER2 gene amplification can be acquired during disease progression or recurrence in $\mathrm{BC}$ patients. ${ }^{31,32}$ Indeed, breast tumor cells may have different pathophysiological states, divide continuously, and harbor the potential for further mutations. In addition, during progression of $\mathrm{BC}$, different clones may coexist as a consequence of increasing genetic instability, and metastatic cells from a HER2-negative primary tumor may undergo amplification during neoplastic spread in an axillary lymph node. On the other hand, the possibility of negative conversion for HER2 in metastatic deposits should not be exclusively attributed to the phenomenon of resistance during trastuzumab therapy, since our patients had not been subjected to neoadjuvant treatment. However, anti-HER 2 therapy may be ineffective in these patients, although it would be worthwhile collecting more information on larger cohorts.

\section{Conclusion}

Our multicenter retrospective analysis confirms that discordance in HER2 status may be present between primary BC 
and synchronous axillary metastatic lymph nodes. Although simultaneous determination of HER2 and hormone receptors in $\mathrm{BC}$ and corresponding metastatic lymph nodes is not mandatory at present, the finding that synchronous metastases from $\mathrm{BC}$ may have a different status when compared with markers from the primary tumor is important. Indeed, this may strongly influence therapeutic management and perhaps impact the prognosis for patients, given that it was demonstrated that loss of HER2 amplification results in worse post-relapse and overall survival in BC patients, ${ }^{13,17}$ and on the other hand, a gain in HER2 expression in metastatic lymph nodes in BC may allow the possibility of a targeted treatment. Thus, our opinion is that significant prognostic information can be obtained from analysis of HER2 status in synchronous metastatic lymph nodes, although further prospective studies are needed to verify the clinical significance of simultaneous assessment of HER2 status in both primary and synchronous metastatic BC.

\section{Acknowledgment}

The skilled statistical support of Professor Giuseppe Giuffrè and his work in preparing tables is gratefully acknowledged.

\section{Author contributions}

$\mathrm{AI}, \mathrm{VB}$, and GT participated in the study conception, design, and acquisition of data, contributed to the interpretation of data, and helped to draft the manuscript. RC, AMC, RDS, DC, PZ, SL, AS, and VF contributed to acquisition and interpretation of the data, and helped to draft the manuscript. All authors read and approved the final paper.

\section{Disclosure}

The authors report no conflicts of interest in this work.

\section{References}

1. Aitken SJ, Thomas JS, Langdon SP, Harrison DJ, Faratian D. Quantitative analysis of changes in ER, PR and HER2 expression in primary breast cancer and paired nodal metastases. Ann Oncol. 2010;21(6): 1254-1261.

2. Chan A, Morey A, Brown B, Hastrich D, Willsher P, Ingram D. A retrospective study investigating the rate of HER2 discordance between primary breast carcinoma and locoregional or metastatic disease. $B M C$ Cancer. 2012;12:555.

3. Hoefnagel LD, Moelans CB, Meijer SL, et al. Prognostic value of estrogen receptor $\alpha$ and progesterone receptor conversion in distant breast cancer metastases. Cancer. 2012;118(20):4929-4935.

4. Aurilio G, Disalvatore D, Pruneri G, et al. A meta-analysis of estrogen receptor, progesterone receptor and human epidermal growth factor receptor 2 discordance between primary breast cancer and metastases. Eur J Cancer. 2014;50(2):277-289.

5. Curtit E, Nerich V, Mansi L, et al. Discordances in estrogen receptor status, progesterone receptor status, and HER 2 status between primary breast cancer and metastasis. Oncologist. 2013;18(6):667-674.
6. Penault-Llorca F, Coudry RA, Hanna WM, Osamura RY, Rüschoff J, Viale G. Experts' opinion: recommendations for retesting breast cancer metastases for HER2 and hormone receptor status. Breast. 2013;22(2): 200-202.

7. Regitnig P, Schippinger W, Lindbauer M, Samonigg H, Lax SF. Change of HER-2/neu status in a subset of distant metastases from breast carcinomas. J Pathol. 2004;203(4):918-926.

8. Santinelli A, Pisa E, Stramazzotti D, Fabris G. HER-2 status discrepancy between primary breast cancer and metastatic sites. Impact on target therapy. Int J Cancer. 2008;122(5):999-1004.

9. Lindström LS, Karlsson E, Wilking UM, et al. Clinically used breast cancer markers such as estrogen receptor, progesterone receptor, and human epidermal growth factor receptor 2 are unstable throughout tumor progression. J Clin Oncol. 2012;30(21):2601-2608.

10. Houssami N, Macaskill P, Balleine RL, Bilous M, Pegram MD. HER2 discordance between primary breast cancer and its paired metastasis: tumor biology or test artefact? Insights through meta-analysis. Breast Cancer Res Treat. 2011;129(3):659-674.

11. Cardoso F, Harbeck N, Fallowfield L, Kyriakides S, Senkus E; ESMO Guidelines Working Group. Locally recurrent or metastatic breast cancer: ESMO clinical practice guidelines for diagnosis, treatment and follow-up. Ann Oncol. 2012;23 Suppl 7:vii11-vii19.

12. Pérez-Fidalgo JA, Eroles P, Ferrer J, et al. An evaluation of the impact of technical bias on the concordance rate between primary and recurrent tumors in breast cancer. Breast. 2013;22(5):974-979.

13. Turner NH, Di Leo A. HER 2 discordance between primary and metastatic breast cancer: assessing the clinical impact. Cancer Treat Rev. 2013;39(8):947-957.

14. Idirisinghe PK, Thike AA, Cheok PY, et al. Hormone receptor and c-ERBB2 status in distant metastatic and locally recurrent breast cancer. Pathologic correlations and clinical significance. Am J Clin Pathol. 2010;133(3):416-429.

15. Solomayer EF, Becker S, Pergola-Becker G, et al. Comparison of HER2 status between primary tumor and disseminated tumor cells in primary breast cancer patients. Breast Cancer Res Treat. 2006;98(2): $179-184$.

16. Liedtke C, Broglio K, Moulder S, et al. Prognostic impact of discordance between triple-receptor measurements in primary and recurrent breast cancer. Ann Oncol. 2009;20(12):1953-1958.

17. Dieci MV, Barbieri E, Piacentini F, et al. Discordance in receptor status between primary and recurrent breast cancer has a prognostic impact: a single-institution analysis. Ann Oncol. 2013;24(1):101-108.

18. Aurilio G, Monfardini L, Rizzo S, et al. Discordant hormone receptor and human epidermal growth factor receptor 2 status in bone metastases compared to primary breast cancer. Acta Oncol. 2013;52(8): 1649-1656.

19. Pusztai L, Viale G, Kelly CM, Hudis CA. Estrogen and HER-2 receptor discordance between primary breast cancer and metastasis. Oncologist. 2010;15(11):1164-1168.

20. Thompson AM, Jordan LB, Quinlan P, et al; Breast Recurrence in Tissues Study Group. Prospective comparison of switches in biomarker status between primary and recurrent breast cancer: the Breast Recurrence in Tissues Study (BRITS). Breast Cancer Res. 2010;12(6):R92.

21. Amir E, Miller N, Geddie W, et al. Prospective study evaluating the impact of tissue confirmation of metastatic disease in patients with breast cancer. J Clin Oncol. 2012;30(6):587-592.

22. Valent A, Penault-Llorca F, Cayre A, Kroemer G. Change in HER2 (ERBB2) gene status after taxane-based chemotherapy for breast cancer: polyploidization can lead to diagnostic pitfalls with potential impact for clinical management. Cancer Genet. 2013;206(1-2): $37-41$

23. Ataseven B, Gologan D, Gunesch A, et al. HER2/neu, topoisomerase $2 \mathrm{a}$, estrogen and progesterone receptors: discordance between primary breast cancer and metastatic axillary lymph node in expression and amplification characteristics. Breast Care. 2012;7(6): 465-470. 
24. Falck AK, Bendahl PO, Chebil G, Olsson H, Fernö M, Rydén L. Biomarker expression and St Gallen molecular subtype classification in primary tumours, synchronous lymph node metastases and asynchronous relapses in primary breast cancer patients with 10 years' follow-up. Breast Cancer Res Treat. 2013;140(1):93-104.

25. Gong Y, Booser DJ, Sneige N. Comparison of HER-2 status determined by fluorescence in situ hybridization in primary and metastatic breast carcinoma. Cancer. 2005;103(9):1763-1769.

26. Simmons C, Miller N, Geddie W, et al. Does confirmatory tumor biopsy alter the management of breast cancer patients with distant metastases? Ann Oncol. 2009;20(9):1499-1504.

27. Perez EA, Suman VJ, Davidson NE, et al. HER2 testing by local, central, and reference laboratories in specimens from the North Central Cancer Treatment Group N9831 Intergroup Adjuvant Trial. J Clin Oncol. 2006;24(19):3032-3038.
28. Niikura N, Ueno NT. Change in HER2 status during breast tumor progression. Cancer Biomark. 2012;12(6):251-255.

29. Richter S, Zandvakili A. Meta analysis of discordant HER2 status in matched primary and metastatic breast cancer. Cancer Res. 2011;71(24):Supplement 3.

30. Jensen JD, Knoop A, Ewertz M, Laenkholm AV. ER, HER2, and TOP2A expression in primary tumor, synchronous axillary nodes, and asynchronous metastases in breast cancer. Breast Cancer Res Treat. 2012;132(2):511-521.

31. Meng S, Tripathy D, Shete S, et al. HER-2 gene amplification can be acquired as breast cancer progresses. Proc Natl Acad Sci U S A. 2004;101(25):9393-9398

32. Kim P, Liu X, Lee T, et al. Highly sensitive proximity mediated immunoassay reveals HER2 status conversion in the circulating tumor cells of metastatic breast cancer patients. Proteome Sci. 2011;9(1):75.
OncoTargets and Therapy

\section{Publish your work in this journal}

OncoTargets and Therapy is an international, peer-reviewed, open access journal focusing on the pathological basis of all cancers, potential targets for therapy and treatment protocols employed to improve the management of cancer patients. The journal also focuses on the impact of management programs and new therapeutic agents and protocols on

\section{Dovepress}

patient perspectives such as quality of life, adherence and satisfaction The manuscript management system is completely online and includes a very quick and fair peer-review system, which is all easy to use. Visit http://www.dovepress.com/testimonials.php to read real quotes from published authors. 